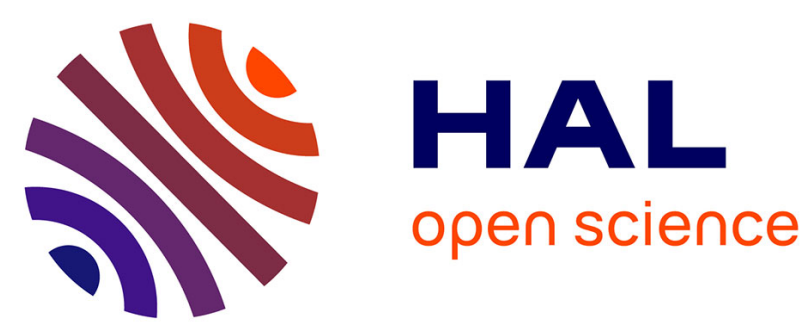

\title{
" Do you feel in control?": Towards Novel Approaches to Characterise, Manipulate and Measure the Sense of Agency in Virtual Environments
}

Camille Jeunet, Louis Albert, Ferran Argelaguet Sanz, Anatole Lécuyer

\section{- To cite this version:}

Camille Jeunet, Louis Albert, Ferran Argelaguet Sanz, Anatole Lécuyer. " Do you feel in control? ": Towards Novel Approaches to Characterise, Manipulate and Measure the Sense of Agency in Virtual Environments. IEEE Transactions on Visualization and Computer Graphics, 2018, 24 (4), pp.14861495. 10.1109/TVCG.2018.2794598 . hal-01679143v2

\section{HAL Id: hal-01679143 \\ https://hal.inria.fr/hal-01679143v2}

Submitted on 22 Jan 2018

HAL is a multi-disciplinary open access archive for the deposit and dissemination of scientific research documents, whether they are published or not. The documents may come from teaching and research institutions in France or abroad, or from public or private research centers.
L'archive ouverte pluridisciplinaire HAL, est destinée au dépôt et à la diffusion de documents scientifiques de niveau recherche, publiés ou non, émanant des établissements d'enseignement et de recherche français ou étrangers, des laboratoires publics ou privés. 


\title{
"Do you feel in control?": Towards Novel Approaches to Characterise, Manipulate and Measure the Sense of Agency in Virtual Environments
}

\author{
Camille Jeunet, Louis Albert, Ferran Argelaguet, Anatole Lécuyer
}

\begin{abstract}
While the Sense of Agency (SoA) has so far been predominantly characterised in VR as a component of the Sense of Embodiment, other communities (e.g., in psychology or neurosciences) have investigated the SoA from a different perspective proposing complementary theories. Yet, despite the acknowledged potential benefits of catching up with these theories a gap remains. This paper first aims to contribute to fill this gap by introducing a theory according to which the SoA can be divided into two components, the feeling and the judgment of agency, and relies on three principles, namely the principles of priority, exclusivity and consistency. We argue that this theory could provide insights on the factors influencing the SoA in VR systems. Second, we propose novel approaches to manipulate the SoA in controlled VR experiments (based on these three principles) as well as to measure the SoA, and more specifically its two components based on neurophysiological markers, using ElectroEncephaloGraphy (EEG). We claim that these approaches would enable us to deepen our understanding of the SoA in VR contexts. Finally, we validate these approaches in an experiment. Our results $(\mathrm{N}=24)$ suggest that our approach was successful in manipulating the SoA as the modulation of each of the three principles induced significant decreases of the SoA (measured using questionnaires). In addition, we recorded participants' EEG signals during the VR experiment, and neurophysiological markers of the SoA, potentially reflecting the feeling and judgment of agency specifically, were revealed. Our results also suggest that users' profile, more precisely their Locus of Control (LoC), influences their level of immersion and SoA.
\end{abstract}

Index Terms-Sense of Agency, Priority Principle, Consistency Principle, Exclusivity Principle, Feeling of Agency, Judgment of Agency, EEG, Neurophysiological Marker, Pre-Motor Cortex, Right Posterior Parietal Cortex, Locus of Control.

\section{INTRODUCTION}

Experiencing a high Sense of Agency (SoA), i.e., feeling in control when performing an action, is essential to ensure the efficiency of an interaction between a user and any technology-mediated application. Indeed, the SoA has been shown to influence the learning performance through the modulation of the technology-acceptance [21]. In other words, it seems that in order to enable learning, the technology should first be accepted by the user, and that the technology-acceptance level depends, inter alia, on the level of control felt by the users when interacting with it.

In Virtual Reality (VR) the SoA is mostly considered as a key element of the Presence phenomenon [37]. Several theories have been developed by the community to characterise the SoA. These theories are centered on the challenges raised by VR, notably on the necessity to trigger the Presence phenomenon and therefore on notions of localisation in space and interaction. This is reflected, inter alia, by the work of Kilteni et al. [24] where the SoA is considered as a dimension of the Sense of Embodiment (SoE) together with the Sense of Self-Location and the Sense of Body-Ownership [24]. Such theories are very relevant for characterising the SoA in the context of VR where it is closely interrelated to notions of body positioning in space. Nonetheless, it is noteworthy that there are other ways to characterise the SoA. Indeed, because the SoA is of great interest for many applications, as well as because its investigation requires notions in human-computer interac-

- Camille Jeunet, Univ. Rennes, Inria, IRISA, CNRS (France) \& CNBI, EPFL (Switzerland). E-mail: camille.jeunet@inria.fr.

- Louis Albert, Univ. Rennes, Inria, IRISA, CNRS (France). E-mail: louis.albert@inria.fr.

- Ferran Argelaguet, Univ. Rennes, Inria, IRISA, CNRS (France). E-mail: ferran.argelaguet@inria.fr.

- Anatole Lécuyer, Univ. Rennes, Inria, IRISA, CNRS (France). E-mail: anatole.lecuyer@inria.fr.

Manuscript received xx xxx. 201x; accepted xx xxx. 201x. Date of Publication xx xxx. 201x; date of current version xx xxx. 201x. For information on obtaining reprints of this article, please send e-mail to: reprints@ieee.org. Digital Object Identifier: $x x . x x x x / T V C G .201 x . x x x x x x x$ tion, psychology and neurosciences, it has been investigated in various disciplines. Notably, literature in cognitive science and philosophy [16] has contributed a lot in the understanding of the SoA. Each of them proposed different and complementary theories and characterisations of the SoA centered on their field of research. In many of these theories, the SoA is considered as a process on its own - and not as a component of a bigger process. Unfortunately, despite the potential benefits of bringing together the different disciplines to study more deeply the SoA, a gap still exists.

As a consequence, the first object of this paper is to contribute to fill this gap by introducing and formalising a theory which could deepen our understanding of the SoA in VR. Among the different theories of the SoA (reviewed in [19]), we chose to focus on a theory stating that the SoA is divided into two components $[15,17,39]$ : the feeling of agency and the judgment of agency, and relies on three principles [45]: the priority principle, the exclusivity principle and the consistency principle. We are convinced that this theory could complement well the theoretical frameworks currently used in VR and provide insights on the elements potentially leading to a decreased SoA in virtual environments.

Based on this theory, novel approaches could be proposed to manipulate the SoA in controlled VR experiments, which could in turn result in new findings and contribute to a deeper understanding of the processes underlying the SoA in virtual environments. In the same vein, by linking this theory to research led in different disciplines such as psychology or neurosciences, we could propose new types of measures of the SoA in VR. More precisely, neuroscientific studies have revealed that the SoA was underlain by specific neurophysiological processes, notably modulations of the amplitude of specific brain rhythms (for a review, see $[10,21,38])$. Most of these studies used intra-cortical measures, Positron Emission Topography (PET) or functional Magnetic Resonance Imaging (fMRI). These methods are not suitable for everyday monitoring of the SoA in VR experiments because all three measures are cumbersome, intra-cortical measures involve a certain risk, and both PET and fMRI are neither portable nor inexpensive. However, it is not clear yet that these processes could be measured using more portable, harmless and cheap brain-measurement techniques such as surface ElectroEncephaloGraphy (EEG) or Near Infra-Red Spectroscopy (NIRS). If it were the case, it would provide a very in- 
novative and powerful tool, that would also provide new perspectives and potentially lead towards a deeper understanding of the SoA. As a consequence, the second main objective of this paper is to propose novel approaches to manipulate and measure the SoA in VR and then to validate these approaches in an experiment. This experiment will allow the investigation of the following questions: Does our approach enable the manipulation of the SoA? Are the 3 principles relevant in $V R$, i.e., is the modulation of each of them associated to a decrease of the SoA? Is it possible to use the EEG to measure the SoA in VR? And more precisely, are there any specific neurophysiological correlates of the SoA -and of its two components- that can be measured using EEG?

Our attempt to formalise the theory, and then propose and validate methods to manipulate and measure the SoA in VR is depicted in this paper, following the subsequent structure. Section 2 is dedicated to a review of the VR literature investigating the SoA. Then, Section 3 aims first to introduce the theory according to which the SoA would be divided into the feeling and judgment of agency and would rely on three principles, and then to justify why this theory is relevant for the VR community. New approaches, based on neuroscience, are then proposed to manipulate and measure the SoA in VR environments in Section 4. These approaches are subsequently tested and validated in Section 5. Finally, a general discussion as well as a conclusion and future works are proposed in Sections 6 and 7.

\subsection{Contributions}

This paper asserts four main contributions:

- Contribution \#1: Introducing and formalising a theory of the sense of agency which aims to deepen our understanding of the SoA in VR. This theory states first that the SoA process could be divided into two components (namely the feeling of agency and the judgment of agency), and second that it relies on 3 principles (namely the principles of priority, exclusivity and consistency).

- Contribution \#2: Proposing an approach to manipulate the SoA, through the modulation of the 3 principles introduced hereinabove, in controlled VR experiments.

- Contribution \#3: Proposing an innovative method to measure the SoA in VR that assesses the feeling and the judgment of agency through their neurophysiological correlates.

- Contribution \#4: Validating the approaches proposed to manipulate and measure the SoA, in a controlled VR experiment $(\mathrm{N}=24)$.

\section{Related Work}

This section aims at briefly introducing the work that has been led to study SoA in VR. First, we will see that from a theoretical standpoint, SoA is defined as a dimension of the more general Sense of Embodiment. Then, we will describe some relevant studies that investigated the behavioral and neurophysiological correlates of SoA in VR.

\subsection{In VR, the sense of agency is considered as one di- mension of the sense of embodiment}

As stated in the introduction, several characterisations of the SoA have been proposed. Here, we chose to focus on a widely accepted one proposed by Kilteni et al. [24]. These authors define the Sense of Embodiment (SoE) as follows: "[A SoE] toward a body B is the sense that emerges when B's properties are processed as if they were the properties of one's own biological body". It is an extension of the definition of De Vignemont [11], who proposed that " $E$ is embodied if some properties of $E$ are processed in the same way as the properties of one's body". Based on the definition of Kilteni et al. [24], the SoE refers to a series of sensations that arise in conjunction with being inside, having, and controlling a body. As a consequence, the SoE can be characterised by the following three components [24]: the Sense of Self-Location, the Sense of Body Ownership and the Sense of Agency.

- The Sense of Self-Location (SoSL) refers to a certain volume in space where one perceives oneself to be located. Rather than being related to the spatial experience of simply being inside the world, the SoSL refers to the spatial experience of being inside a body. It is thus highly determined by the egocentric visuospatial perspective. SoSL can be experienced in Virtual Reality using a first person perspective, i.e., by respecting the position of the eyes of the artificial body. A third person perspective, conversely, will break the natural conditions in which subjects experience self-location with respect to their real body [12].

- The Sense of Body Ownership (SoBO) refers to the sense that "I am the one who is undergoing an experience" [16], or to the fact that "one's own body is the source of sensation" [41]. In virtual reality, the SoBO can be increased by creating sensory (e.g., visuotactile or visuoproprioceptive) correlations between one's biological body and the seen stimulation on the virtual body. This principle underlies the well-known rubber-hand illusion [7]. Globally, on both structural and morphological aspects, the more similarities between one's biological body and the virtual body, the more SoBO perceived.

- The Sense of Agency (SoA) is the sense that "I am the one who is causing or generating an action" [16]. More precisely, it refers to the sense of having "global motor control, including the subjective experience of action, control, intention, motor selection and the conscious experience of will" [4]. The sense of agency can be induced in virtual reality when the motion of the participant is exactly replicated in real-time (or near real-time) on the virtual body. More details about the SoA, the ways to modulate it and its neural correlates are provided in Section 4.1.

While questionnaires are most of the time used to assess the different dimensions of the SoE, their specific neurophysiological correlates have also been investigated in VR. Due to the popularity of the "rubber-hand illusion" the majority of these studies have investigated the correlates of the body-ownership and self-consciousness dimensions [5, 14, 18, 42] The neurophysiological correlates of the sense of self-location have also been investigated, albeit more modestly $[2,9,29]$. In this paper, we will focus specifically on the SoA dimension, which may be the one that has been the least investigated by the community so far.

\subsection{Investigations of the sense of agency and its neuro- physiological correlates in virtual reality}

\subsubsection{Investigating the SoA in VR using behavioural measures}

Argelaguet et al. [1] investigated the impact of the realism of operators' hands on their SoA. Thus, they implemented a pick-and-place task in a virtual environment and asked participants to perform this task with different kinds of virtual hands. Their results show that the SoA was higher with less realistic hands for which there was less of a mismatch between participants' real actions and the animation of the virtual hand. Nonetheless, the sense of body-ownership was higher with the humanlike virtual hand. Furthermore, in their study Tieri et al. [40] assessed operators' sense of body-ownership and SoA during passive movement observation of an avatar both when the full limb was displayed (here, the full arm) and when there were "visual discontinuities" (i.e., absence of some segments of the limb, between the wrist and the elbow). Their results suggest that passive observation can indeed induce a SoA and a sense of body-ownership but that they are both impaired in case of visual discontinuities. These results are consistent with the ones of Kokkinara et al. [26] who have shown that a seated person immersed in a virtual environment with a first person perspective could experience an illusory SoA over a walking avatar. Nonetheless, this SoA significantly decreased in the third-person perspective condition. Kokkinara et al. [27] also investigated the effects of spatial (angular offset) and spatiotemporal (modification of the speed) distortions of the visual feedback in a virtual environment on operators' SoA and SoBO. Therefore, they implemented a task in which participants had to reach a target (a semi-transparent ball). Either the speed of their movement or the angular offset of their arm was increased when replicated on the avatar. Results show that both of these modifications changed the 
participants' proprioceptive judgment of the size of the objects but did not alter their SoBO. However, the SoA dropped significantly in case of spatiotemporal distortions. Finally, Banakou et al. [3] investigated how VR users tend to attribute an action to themselves in a virtual environment. Thus, they embodied participants in an avatar and placed them in front of a mirror in a virtual environment. For half of the participants, the avatar moved synchronously to themselves while for the other half, the avatar's actions were uncorrelated to their own actions. The avatar spoke with corresponding lip movements and half of the participants received in addition vibrotactile stimulations on the thyroid cartilage. Results show that participants in the synchronous condition tended to attribute the speaking to themselves. The authors argue that the sense of body-ownership plays a crucial role in modulations of the SoA, which could somehow be in opposition with Kalkert and Ehrsson [22] results which had suggested a double dissociation between the sense of body-ownership and the SoA, and thus the independence of both processes. These results, while insightful, show that we are still far from a full understanding of the SoA process. This may be explained, at least in part, by the fact that so far questionnaires have mainly been used to assess operators' SoA. While such questionnaires have the advantage of being simple to use, they also present some flaws: among others, the scores are likely to be dependent on the context, i.e., on the environment (e.g., observer-expectancy effect) and on the cognitive and conative states of the operator (e.g., fatigue, level or attention or motivation). Thus, while very insightful, the scores obtained at questionnaires can variate along the experiment due to confounding factors, independently from the level of agency. They also require the operator to perform an additional task (completing the questionnaire) and thus do not enable "real-time/in-task" recordings of the level of agency. Therefore, using additional measurement techniques, overcoming these flaws, could be of the greatest interest. Specific physiological and neurophysiological measurements present the advantage of being less subject to confounding factors and of enabling "real-time/in-task" recordings. Such neurophysiological correlates have been investigated in some VR studies. They are introduced in the next section.

\subsubsection{Investigating the SoA in VR using neurophysiological measures}

Nahab et al. [33] carried out an ecological virtual reality experiment during which participants had to perform finger movements. The visual feedback they received was more or less manipulated, i.e., by moving their hand, participants were controlling the virtual hand movement at $100 \%$ (full agency), $75 \%, 50 \%, 25 \%$ or $0 \%$ (no agency). The behavioural results suggested the existence of a bias that led subjects to over- or underestimate the amount of control they had. Then, the neurophysiological results - obtained using fMRI recordings - suggest that the right supramarginal gyrus, left anterior inferior parietal lobule, anterior insula, and right temporoparietal junction might be involved in mismatch identification, and that the bilateral prefrontal, cingulate, and bilateral posterior inferior parietal lobule might process this mismatch information and consequently generate an appropriate SoA [33]. A following EEG study [23], performed using the same experimental protocol, suggested that alpha band activity may be "the main neural oscillation" involved in the SoA and that the neural network within the anterior frontal area may be important in the generation of SoA [23]. Furthermore, Padrao et al. [35] investigated the neurophysiological correlates of violations of agency. Indeed, such violations, that correspond to a mismatch between the expected and actual outcome of one's actions, can be detected through the triggering of error detection mechanisms. The authors investigated these errors mechanisms by asking the following question: are the error-detection mechanisms the same for self-generated and external actions? Their results show a clear neural dissociation between both processes. Indeed, while "real errors" (i.e., self-generated errors) elicited a classic error potential, namely an N100 in fronto-central areas, "false errors" (i.e., errors generated by the avatar) elicited a parietal N400 which typically characterises semantic or conceptual violations. In addition, the amplitude of the N400 correlated with the subjective feeling of body-ownership.

This section provided an overview of the studies led in VR to invest- igate the SoA. In the next section, we propose a characterisation of the SoA that could enable us to further understand this process.

\section{INTRODUCING THE TWO COMPONENTS AND THREE PRIN- CIPLES ON WHICH THE SENSE OF AGENCY RELIES}

\subsection{Theoretical aspects - The Sense of Agency has $2 \mathrm{com}$ - ponents and relies on 3 principles}

\subsubsection{Defining the Concept of Sense of Agency: from the Feel- ing of Agency to the Judgment of Agency}

As stated earlier, the Sense of Agency (SoA) corresponds to the sense that "I am the one who is causing or generating an action" [16]. This SoA can be divided into 2 components [15, 17,39]: (1) the feeling of agency and (2) the judgment of agency. These two concepts of feeling and judgment of agency correspond to what Banakou and Slater [3] present as one factor explaining the SoA, the "cause (intent to act) preceding the effect (the results of the action)". The feeling of agency is pre-reflective, implicit, low-level and non-conceptual while the judgment of agency is reflective, explicit, high-order, belief-like and conceptual. In other words, the feeling of agency precedes the action outcome (i.e., the perception of the feedback), it is triggered at the very early stages of the action, while the judgment of agency results from the computation of the comparison between the predicted and actual outcomes of the action (i.e., it is computed once the feedback has been perceived and processed). Synofzik et al. [39] explain that a feeling of agency must be conceptually processed for a judgment or an attribution of agency to occur. In order to experience a judgment of agency, three principles must be respected [45] when providing the feedback. These principles are described in the next section.

\subsubsection{Introducing the 3 Principles Conditioning the Agency}

The judgment of agency relies on three principles, which would be the general conditions for attribution of causality [45]:

- The priority principle: the conscious intention to perform an act must immediately precede the action, which in turn should immediately precede the outcome.

- The consistency principle: the sensory outcome must fit the predicted outcome.

- The exclusivity principle: one's thoughts must be the only apparent cause of the outcome (i.e., one must not believe there to be an outside influence)

While raised and discussed in some VR papers, notably in [3], to our knowledge these three principles have never been formally considered so far in VR to study the SoA. Furthermore, in addition to these three principles several indicators influencing the judgment of agency have been proposed $[43,44]$ : bodily and environmental cues ("Where am I?"), bodily feedback (proprioceptive and kinesthetic information), bodily feedforward (i.e., the predicted sensory feedback), sensory feedback, social cues, action consequences and action-relevant thoughts (thinking about doing beforehand, in other words, the feeling of agency). On the one hand, the absence of some of these indicators can lead to "a case of automatism" [43], that is to say to the absence of judgment of agency: the agent is "doing without feeling". On the other hand, the manipulation of the same markers can lead to "an illusion of agency/ownership" [43]: agents who are "feeling without doing", and thus think they are in control although they are not.

Manipulating of disregarding these principles and elements (i.e., principles and indicators introduced hereinabove) can thus potentially impair VR users' sense of agency. In the following section, we will introduce some examples of situations in which the sense of agency can be impaired in VR environments, by targeting more specifically the "3 principles" introduced hereinabove as they are the central focus of this paper. 


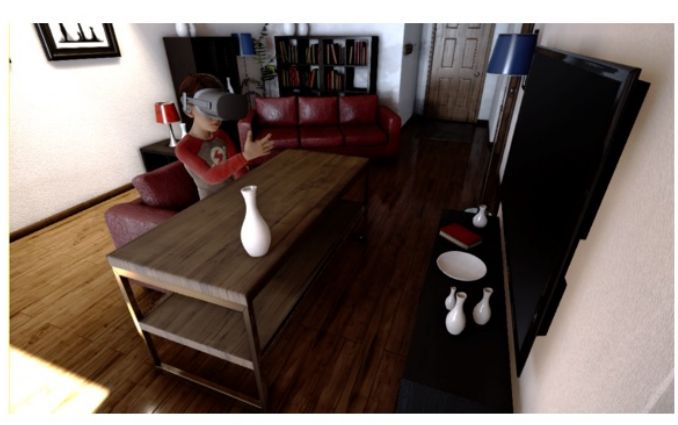

(a)

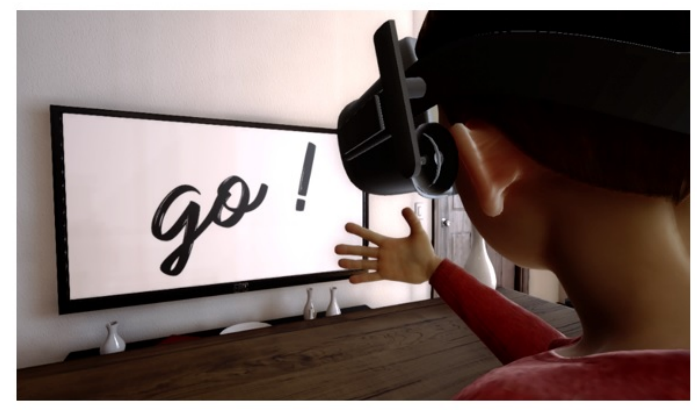

(b)

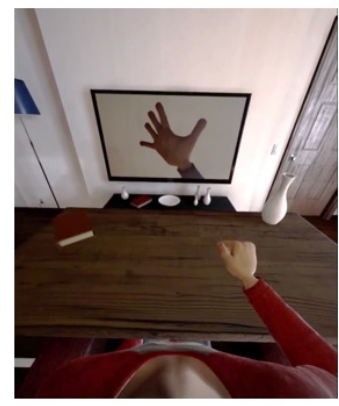

(c)

Figure 1. (a) Overview of the VR scene; (b) Third-person perspective: the participant receives a go signal and starts to perform the movement; (c) First-person perspective: the participant is provided with an instruction through a video displayed on the virtual screen (once the video ended, the go picture will tell them to start moving).

\subsection{Practical aspects - The manifestation of these prin- ciples in Virtual Reality systems}

By definition, virtual environments are simulated. Despite the great improvement of computational power, tracking systems precision and response, and head-mounted displays quality, virtual environments can still suffer from technical limitations. In this section, we advocate that such limitations can potentially induce modulations of the three principles introduced hereinabove, in turn resulting in a decreased SoA.

First, low responsive tracking systems as well as simulations requiring too much computation (e.g., due to high quality rendering) can introduce latency. In other words, the time between the action performed by the user and the consequent update of the virtual environment is extended. Thus, the feedback does not anymore immediately follow the action, which modulates the priority principle and thus could theoretically result in a decreased SoA.

Second, jumps in the tracking can occur owing to network issues or occlusions of the tracker to name only a few. In such cases, the latest movements of the operator will not be taken into account for the visual feedback in the virtual environment. Instead, previous positions of the limbs will be displayed until the tracking works again. In such circumstances, the sensory feedback is different from the real action and thus from the predicted feedback, which could result in a modulation of the consistency principle and in a potential reduction of the SoA.

Finally, the precise replication of the operator's movements in a virtual environment requires this operator to be equipped with numerous sensors. Most of the time, technical constraints prevent the operator from being equipped with enough sensors in which case extrapolations are required. Thus, based on physical, anatomical or behavioural models, algorithms will be used to extrapolate the movements of the body parts not equipped with sensors, from the movements recorded on the equipped limbs. However, such algorithms can make errors of prediction and, for instance, assume that a body-part moved while it did not. In this case, the operator's thoughts are not anymore the only apparent cause of the outcome. The exclusivity principle is modulated, which may induce a drop of the operator's SoA.

All together, these examples illustrate the potential gains in considering these 3 principles in order to better understand how and why the SoA is impaired in some virtual environment. Now, in order to experimentally evaluate the relevance of these principles, specific approaches to manipulate them and then consequently measure the SoA should be proposed. This is the object of the next section.

\section{Novel approaches to manipulate and MEASURE the SENSE OF AGENCY IN VIRTUAL ENVIRONMENTS}

\subsection{Manipulation of the sense of agency}

The object of this section is to propose an approach to manipulate each of the three principles in a controlled experiment (aiming, for instance, at investigating the sense of agency in VR). We chose to focus on hand movements for several reasons. First, they are the main body medium for interaction in VR environments. Also, given their high number of degrees of freedom, they enable the user to perform a lot of different kinds of movements and are therefore often used by VR operators to test the responsiveness of VR environments.

As a matter of fact, we hereafter propose a manipulation of the SoA based on the modulation of the three principles described in Section 3.1.2. For each of the principles, we propose three modalities of modulation in order to avoid too much habituation.

- Priority principle (the conscious intention to perform an act must immediately precede the action, which itself must immediately precede the outcome): in order to modulate the SoA based on this principle, we propose to add a visual latency between the user's movement and the feedback (i.e., the virtual hand's movement). Here, we propose this latency to be of either $1 \mathrm{~s}, 1.5 \mathrm{~s}$ or $2 \mathrm{~s}$.

- Consistency principle (the sensory outcome must fit the predicted outcome): here, we propose to manipulate the feedback through the inversion of two fingers in order to modulate the consistency principle. For instance, the virtual hand's index could move as the real hand's ring finger and vice-versa. These inversions would include proximal, intermediate and distal phalanges. In order to increase the visual discrepancy, and thus the likeliness that this inversion is perceived by the operator, we propose not to inverse the movements of adjacent fingers but rather of more distant fingers. Also, the thumb axis being different from the axes of the other fingers, a movement inversion between the thumb and another finger is more complex to compute while aiming at maintaining a realism in the movements. As a consequence, we propose inversions of either the index with the ring finger, the index with the little finger or the middle with the little finger.

- Exclusivity principle (one's thoughts must be the only apparent cause of the outcome): finally, in order to modulate the exclusivity principle, we propose to make one of the fingers not responsive to users' movements. In other words, this finger would "move by itself". This "mad finger" could be any of the fingers. Here, we propose to select the thumb, the middle and the little fingers.

\subsection{Measure of the Sense of Agency: Taking advantage of the research in neuroscience}

In Section 2.2, we presented some VR studies investigating the neurophysiological correlates of the SoA. This section aims in turn to bring forward studies led by other communities that revealed neurophysiological correlates of the SoA potentially underlying the theory we propose, notably the fact that the SoA is divided into two different components.

Several studies have been investigating the neurophysiological correlates of the SoA. Reviews of these correlates have been proposed in $[10,21,38]$. To summarise, despite the fact that the neural correlates 
of the SoA remain barely understood [14], some brain areas have been suggested, quite consistently over different studies, to be involved in the fact of feeling or not agent. Self-agency has been shown to be underlain by an increased activity in the Pre-Motor Cortex (PMC) $[14,15]$ and more specifically in its ventral part, the Supplementary Motor Area (SMA) [15,28]. In Farrer and Frith [15], it has also been suggested that an increase of the activity of the anterior insula occurred when a person was aware of causing an action. Contrariwise, the activation of the right Posterior Parietal Cortex (PPC) has been shown to negatively correlate with the SoA: the more a person tends to attribute the action to another person, the more the PPC is activated [15]. In other words, the activity in the PPC increases when there is a mismatch between the predicted and the actual sensory outcomes of the action [8]. Also, the cerebellum would act as a relay to inform about the sensorimotor discrepancies between the predicted and actual outcomes of the action [10]. While consistent, these correlates are still discussed. For instance, in [28], no correlation between the PPC activation and their subjective measure of agency was reported.

As a reminder, we proposed to characterise to SoA as a process that can be divided into 2 components: the feeling of agency and the judgment of agency. On the one hand, the pre-motor cortex being solicited at very early stages of the action, we hypothesise that its activation could specifically reflect the "feeling of agency" component. On the other hand, the fact that the right posterior parietal cortex is activated when there is a mismatch between the predicted and the actual sensory outcome suggests that its degree of activation may reflect the "judgment of agency" component.

These correlates have essentially been revealed thanks to the use of intra-cortical EEG or fMRI measures. Because we cannot reasonably rely on these brain imaging techniques for wide-spread studies of the SoA, we propose here to investigate the feasibility of using surface EEG to detect these specific markers of the SoA, and more specifically of its two components. NIRS also has the advantage of being portable and cheap together with having a high spatial resolution. Therefore, its combination with EEG, which has a best temporal resolution (and thus seems to be the best candidate for this study), could be investigated in future works. We used a 32-channel EEG cap in order to have a high-density coverage of the fronto-central (around the pre-motor cortex) and parietal areas. We propose to record the EEG activity both in high-agency and low-agency conditions, low-agency conditions being obtained through the modulation of the three principles. Based on Haggard [19], who states that "the key neural correlate of sense of agency might lie in the connectivity between frontal and prefrontal motor areas that initiate action and parietal areas that underlie the monitoring of perceptual events", the amplitude of the signals recorded should be higher over the pre-motor cortex at early stages of the action in the high-agency condition (i.e., when the principles are not modulated), while it should be higher over the right posterior-parietal cortex when the feedback is perceived in the low-agency condition (i.e., when one of the principles is modulated).

\section{EXPERIMENTAL VALIDATION OF OUR APPROACHES TO MA- NIPULATE AND MEASURE THE SENSE OF AGENCY IN VIR- TUAL ENVIRONMENTS}

\subsection{Rationale and hypotheses}

This paper aims to introduce new approaches to characterise, manipulate and measure the SoA in virtual environments with the long term objective of deepening our understanding of this process and improve VR systems. Thereby, the following experiment aims at testing the relevance of our approach, and more precisely to answer the following questions: Is this characterisation relevant in VR? Does the modulation of each of the three principles induces a decrease of the SoA? Are there specific EEG markers of the SoA and more specifically of its two components?

Concerning the manipulation of the SoA, the approach to modulate the three principles introduced in Section 4.1 will be applied. We hypothesise that the perceived SoA (which will be measured using questionnaires) will be higher when the principles are not modulated than when they are. Concerning the measure of the SoA, we will use

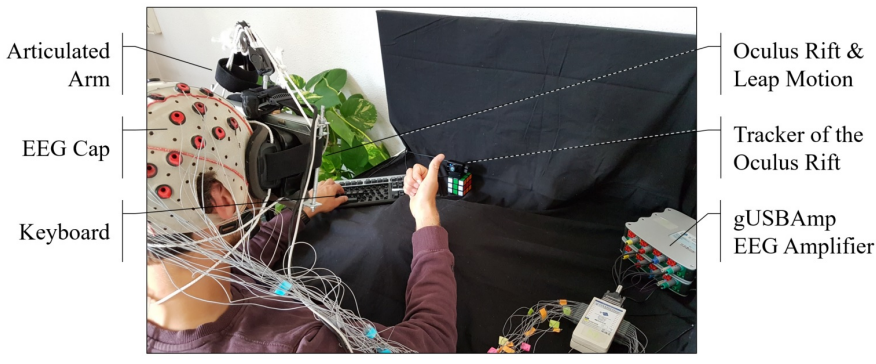

Figure 2. Photo of the experimental set-up. The participant is equipped with an EEG cap, plugged to g.USBAmp amplifiers. In addition, he is immersed in the virtual environment using an Oculus Rift attached to his head and supported by an articulated arm (to avoid any pressure on the EEG cap and reduce the risk of muscular fatigue). Finally, his head is tracked by the Oculus tracker and his right hand is tracked by a Leap Motion fixed in front of the Oculus Rift.

(in addition to the hereinabove mentioned questionnaires) the EEG for the reasons explained in Section 4.2. We hypothesise that a stronger activation of the pre-motor cortex, reflecting a high feeling of agency, will be revealed at the early stages of the trials in "high-agency" conditions (i.e., when the principles are not modulated), while a stronger activation of the right posterior-parietal cortex, reflecting a low judgment of agency, will be revealed after the feedback is perceived in "low agency conditions" (i.e., when one of the principles is modulated).

\subsection{Participants}

24 naive participants ( 5 women, 19 men; aged $23.54 \pm 2.84$ year-old) volunteered to take part in this study. They were all right-handed according to the Test of Laterality of Harris [20]. They all completed and signed an informed consent form at the beginning of the session. The experiment procedure conformed to the Declaration of Helsinki.

\subsection{Experimental procedure}

The experiment consisted in a 2 hour-long session during which participants had to rate their perceived level of agency (from 1 to 7 - Likert scale) right after performing some specific movements of the right-hand in a virtual environment. Their brain-activity was recorded during the task and processed offline in order to investigate the potential neurophysiological correlates of the SoA. Hence, at the beginning of the session, participants first completed an informed consent form, a basic information questionnaire (age, gender, number of hours of sleep) as well as the Test of Laterality of Harris [20] that enabled the experimenter to check that each participant was right-handed. Then, they completed the "Internal - Powerful Others - Chance" (IPC) cognitive test [30]. This 24-question questionnaire aims to determine participants' LoC (i.e., if they mainly consider the results of their actions as due to themselves, to powerful people or to chance) based on questions related to their perception of the outcomes of everyday situations. Among others, the LoC has been shown to correlate with the sense of presence [32]. As a consequence, we were willing to investigate its relationship with agency. Once this step completed, the 32-electrode EEG cap was installed and participants were read the instructions for the following experiment. All participants were provided with exactly the same instructions, which indicated to them that they would have to perform 3 runs (sequences) of around 12 minutes each, during which they would be immersed in a virtual environment (see Figure 1): a living room, equipped with a TV on which some pictures and videos would be displayed, and a table on which the virtual hand of their avatar would be placed. Each of these runs started with a 30s-long baseline, and was then divided into 9 blocks of trials. One block of trials was structured as follows: first, a video, displayed on the virtual TV and used as the instruction, presented one of the 3 pre-determined hand-movements (counting, tapping or abducting/adducting the fingers - see Figure 3) for 4 seconds; following this video, participants had to perform the demonstrated movement repeatedly for 6 trials of 8 seconds each. Each 


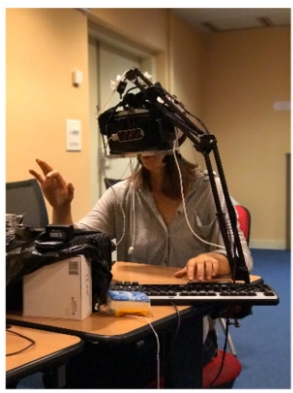

Tapping

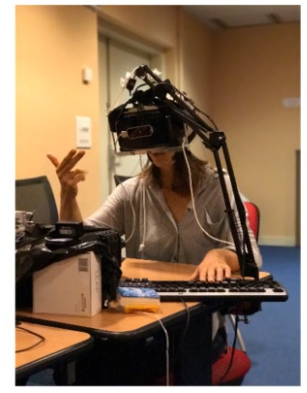

Counting

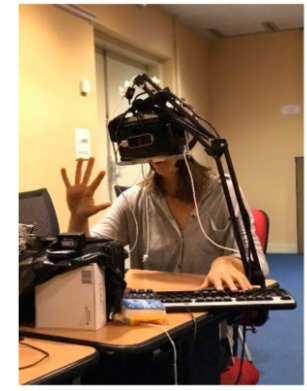

Adducting \& Abducting

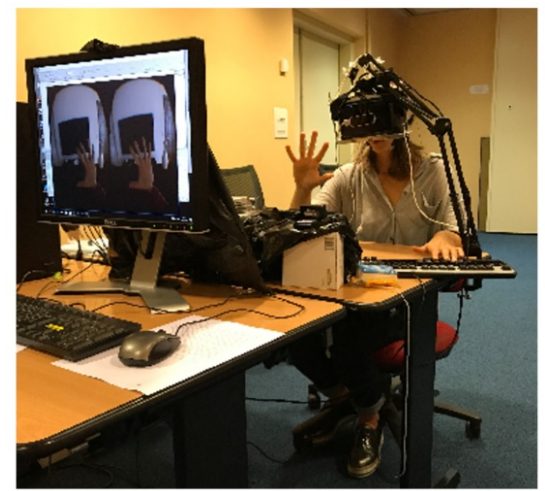

Figure 3. Photos representing a participant performing the 3 kinds of pre-determined movements: tapping, counting and adducting/abducting the fingers. On the far-right photo, we can also observe what the participant see in through the HMD. In this case, the feedback is not manipulated, so she is provided with a visual feedback replicating her real movements.

trial started with a "GO" picture displayed on the virtual TV. After 8s, a "STOP" picture indicated the participant they could stop performing the movement, and the following question was asked: "On a scale ranging from 1 to 7 , how much did you feel in control during this trial?". Participants had to press the appropriate key (between 1 and 7) on the keyboard and then to validate by pressing the space bar. The next block of trials started 1s afterwards. Among the 6 trials of one block, 3 were not manipulated while the other 3 were manipulated, each of them following a different principle, i.e., the priority, consistency or exclusivity principle. In total, the SoA could strictly be modulated in 9 different ways. In total, participants performed 162 trials ( 3 runs $* 9$ blocks $* 6$ trials) among which half were manipulated. Indeed, each of the 3 principles can be modulated in 3 different ways, e.g., the priority principle has 3 modalities: a lag of $1 \mathrm{~s}, 1.5 \mathrm{~s}$ and $2 \mathrm{~s}$. Nonetheless, as the different modalities of each principle were not meant to be associated with different levels of SoA, they were considered the same for the analyses. The movement associated with each block of trials as well as the order of the trials (manipulated and not-manipulated) within the blocks and the modality of the modulation were counterbalanced between the participants in order to avoid any order effect. The only parameter that remained constant was the first trial of each block: it was always a not-manipulated, i.e., supposedly high agency trial. This way, the score of perceived agency provided at this trial served as a reference for the other trials of the block. After 9 blocks of trials, the run was over. The VR apparatus was temporally taken off and participants were proposed a break before they started the next run. Finally, at the end of the session, they had to complete a "Final Questionnaire" (inspired from Argelaguet et al. [1]) the goal of which was to assess their global level of immersion.

\subsection{Experimental set-up}

A major challenge consisted in measuring participants' EEG (using a 32 channel device, with electrodes covering the whole scalp area) at the same time they were immersed in a virtual environment, through the use of a Head-Mounted Display (HMD) device. Indeed, while no pressure should be applied on the electrodes (to avoid any distortion of the recorded signal), HMD devices are usually maintained to users head using elastic bands placed around the head. We thus had to design a system that enables the HMD to be fixed to the head without using elastic bands. We decided to make use of an articulated arm on which the HMD was attached. The user could place their head on the HMD which was attached to them using a single string of rope. In addition to its advantage of not applying any pressure on the electrodes, this system enabled participants to have some mobility of the head and to support the weight of the HMD device, thus reducing the muscular fatigue. The physical configuration of the experiment is shown in Figure 2.

For immersing participants in our virtual environment we used the Oculus Rift (DK2) HMD, which has a resolution of 960 px x 1080 px per eye and a $100^{\circ}$ nominal field-of-view. Participants were seated in front of a table on an adjustable (height, backrest and seat) office chair to allow them to have a comfortable position during the experiment. The head tracking was provided by the Oculus Rift (DK2), the tracker of which was positioned on the table in front of the participant, on the left, to avoid any obstacle between the Oculus Rift and its tracker (such as the hand during movements) which could cause jumping video streaming due to loss of tracking. Also, a small box was placed on the physical set-up in front of the participant in order to elevate their right hand and thus to maximise its position in the field of view of the Leap Motion / Participant and to avoid a potential muscular fatigue.

The graphics were generated by a desktop computer equipped with an Intel Xeon E5-1603 v4 processor and 16Gb Random-Access Memory. The PC had one Nvidia GeForce GTX 1080 graphic card. This PC ensured a constant $75 \mathrm{~Hz}$ display rate during the experiment. The participants' hands were tracked using a Leap Motion. The Leap Motion was placed on the Oculus Rift using the Leap Motion Universal VR Developer Mount. It allowed a permanent interaction space in front of the head of the participant, even when they moved their head. The environment in front of the participant was covered by an anti-reflective tissue to limit infra-red interferences and ensure optimal tracking. The Leap Motion SDK used was the latest available (Orion). The virtual hand was replicating the participants' movements in near real time $(\leq 50$ ms latency). The virtual environment used in the experiment matched the physical set-up providing both a reference frame and passive haptic feedback when touching the desktop. A scheme of the experimental set-up is proposed in Figure 2.

The experiment was implemented in Unreal Engine 4.15.3 using both $\mathrm{C}++$ and blueprint (Visual Scripting system of Unreal Engine). The virtual environment was based on the Unreal Engine Realistic Rendering Technical demo. To avoid the Uncanny Valley effect and maximise the SoA while keeping a morphological and human like virtual avatar, we used a semi-realistic/semi-cartoon virtual avatar. Indeed, the SoA was demonstrated to be kept even when avatars are not realistic [46] or differ from the users morphology [25,31]. The use of a cartoon-like character enabled us to avoid having to adapt the shape of the hand and skin colour to each participant, as we would have had to do if the representation had been more realistic. Details about the EEG set-up, recording and pre-processing are provided in the next section.

\subsection{Recording and Pre-Processing of the EEG Data}

The EEG was recorded using two g.USBAmp amplifiers (g.tec, Austria), by means of 32 wet (g.tec LadyBird) scalp electrodes (C6, CP5, CP1, CP2, CP6, P7, P5, P3, Pz, P4, P6, P8, PO7, PO3, PO4, PO8, F5, F1, F2, F6, FC5, FC3, FC1, FC2, FC4, FC6, C5, C3, C1, Cz, C2, $\mathrm{C} 4,10-20$ system), referenced to the right ear and grounded to AFz. Such electrodes cover the whole scalp, with a higher density above the pre-motor and parietal areas, which are our main areas of interest. Indeed, as described in Section 4.2, modulations of the SoA would be underlain by specific modulations of the EEG activity in the pre-motor 
Perceived Agency Score as a function of the Manipulation Type \& Run.

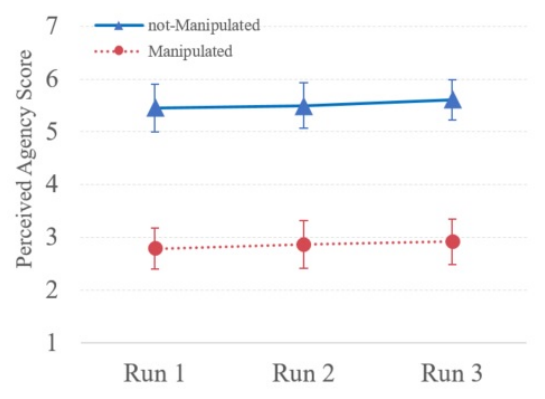

Perceived Agency Score as a function of the Modulation Type (Priority, Consistency, Exclusivity) \& Run.

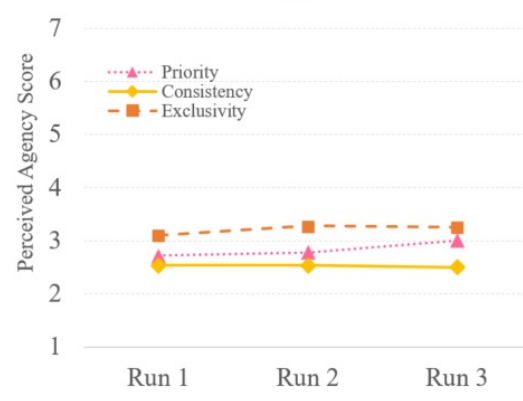

Figure 4. Diagrams representing the perceived agency score as a function of the condition and of the run. On top, the average scores obtained at the manipulated vs. not-manipulated trials are displayed (with standard deviation). The 2-way ANOVA for repeated measures revealed a main effect of the Manipulation as well as a main effect of the Run. Down, the scores provided for each modulation type are displayed. Here, the 2-way ANOVA for repeated measures revealed a main effect of the modulation type. Ad-hoc analyses indicate that modulations of the priority principle were associated with better scores that the other 2 .

and parietal cortices. EEG data were sampled at $512 \mathrm{~Hz}$. For the offline analyses, EEG signals were band-pass filtered in $1-40 \mathrm{~Hz}$ and epoched. The epochs started $1000 \mathrm{~ms}$ before the start of the trial (i.e., the "GO" picture) and ended $8000 \mathrm{~ms}$ after the start of the trial (i.e., when the "STOP" picture was displayed). Then, a semi-automatic method was used for artefact removal: using EEGLab, epochs containing abnormal values (inferior to $-200 \mu \mathrm{V}$ or superior to $200 \mu \mathrm{V}$ ) or abnormal trends (maximal slope superior to $200 \mu \mathrm{V}$ with a $\mathrm{R}$-squared limit fixed to $0.3)$ were first marked down. Then a visual inspection of all the data sets enabled the rejection of artefacted epochs. Then, data were rereferenced using Common Average Referencing (CAR). Finally, a baseline removal algorithm was applied, using the $200 \mathrm{~ms}$ preceding the "GO" picture period as baseline. The data were recorded using OpenViBE [36] and pre-processed using Matlab/EEGLab [13].

\subsection{Results}

The SPSS software [34] was used to perform the behavioural statistical analyses, while the neurophysiological analyses (statistics and visualisations) were performed using Matlab and the EEGLab Plug'In [13].

\subsubsection{Behavioral results}

Manipulation of the Agency: A 2-way ANOVA for repeated measures considering the Run $\left(\mathrm{R}_{3}\right.$ : run 1 vs. run 2 vs. run 3$)$ and the Manipulation ( $\mathrm{M}_{2}$ : manipulation vs. no manipulation) as factors was performed to investigate the effectiveness of our manipulations to modulate users' SoA. Thus, the dependent variable was the average perceived agency score, self-reported after each trial. The normality assumption was respected, based on the Shapiro-Wilk test. However, the sphericity assumption was violated for the Run*Manipulation interaction ( $\mathrm{p}=.012$ ) according to the Mauchy's sphericity test. Thus, we corrected the degrees of freedom (using the Huynh-Feldt correction) when reporting the results of this analysis. The scores attributed after each trial indicate that we managed to manipulate participants' agency. The perceived agency scores were significantly lower in the manipulated trials (modulation of the priority, consistency or exclusivity principles) than in the non-manipulated trials. The ANOVA indeed revealed a main effect of the Manipulation $\left[\mathrm{F}(1,23)=323.537, \mathrm{p} \leq .001, \eta^{2}=0.934\right]$. It also revealed a main effect of the Run $[F(1,23)=4.626, p \leq .05$, $\left.\eta^{2}=0.167\right]$ but no Run*Manipulation interaction $[\mathrm{F}(1.582,46)=0.155$, $\left.\mathrm{p}=.808, \eta^{2}=0.007\right]$. Indeed, post-hoc analyses revealed a slight but still significant increase of the Agency scores between Run 1 and Run $3[$ agencyR $1=4.11 \pm 0.76$, agencyR $3=4.26 \pm 0.69 ; \mathrm{t}(23)=2.151$, $\mathrm{p} \leq .05]$ (see Figure 4, up).

Furthermore, the average perceived agency score positively correlated with the level of immersion computed from the responses to the Final Questionnaire (average score of all the items of the questionnaire) $[r=0.473, p \leq .05]$. A positive correlation was also found between the "Intern" dimension of the IPC and the level of immersion (computed from the responses to the Final Questionnaire) $[r=0.473, p \leq .05]$ (see Figure 5, down) as well as with the perceived agency scores in the non-manipulated trials $[\mathrm{r}=0.433, \mathrm{p} \leq .05]$ (see Figure 5, up). These results suggest that the "Intern" dimension of the Locus of Control influences VR users' basic level of agency as well as their feeling of immersion. More precisely, participants with a high "Intern" score, who theoretically attribute the cause of the feedback they receive mainly to themselves (rather than to the device, other people or chance), have a higher basic level or agency and feel more immersed even if the feedback they are provided with is manipulated.

Effect of the Type of Modulation on the Perceived Agency: In order to determine the specific effect of the different types of modulations on the perceived agency, a 2-way ANOVA for repeated measures was performed, with the Run $\left(\mathrm{R}_{3}\right.$ : run 1 vs. run2 vs. run 3$)$ and the Modulation-Type $\left(\mathrm{M}_{3}\right.$ : priority vs. exclusivity vs. consistency) as factors. This analysis principally revealed a main effect of the Modulation-Type $\left[\mathrm{F}(1,23)=4.899, \mathrm{p} \leq .05, \eta^{2}=0.176\right]$. Post-hoc analyses indicated that the perceived agency in the case of a modulation of the exclusivity principle was significantly higher than when the priority $[\mathrm{t}(23)=2.213, \mathrm{p} \leq .05]$ or consistency $[\mathrm{t}(23)=8.845$, $\mathrm{p} \leq .001]$ principles were modulated, see Figure 4 , down. Nonetheless, we should stay cautious when interpreting these comparisons as we do not have any formal metric enabling the comparison of the level of manipulation of the agency generated by the different modalities of each principle. In other words, the differences of perceived agency could be due to the modalities selected rather than to the principles themselves. However, most importantly, all the Modulation-Types seem to be efficient to manipulate users' SoA. Indeed, the average score of perceived agency was significantly lower in the manipulated trials (i.e., supposed low agency conditions) than in the non-manipulated trials (i.e., supposed high agency conditions), as suggested by the ttests for paired samples: non-manipulated vs. Priority [t(23) $=14.191$, $\mathrm{p} \leq .001]$, non-manipulated vs. Exclusivity $[\mathrm{t}(23)=15.996, \mathrm{p} \leq .001]$ and non-manipulated vs. Consistency $[\mathrm{t}(23)=17.554, \mathrm{p} \leq .001]$.

\subsubsection{Neurophysiological results}

First, it should be noted that 4 participants had to be excluded from the neurophysiological analyses due to inoperable EEG data (too many artifacts). The following analyses were thus performed on the remaining 20 participants. As a reminder, data were first pre-processed as explained in Section 5.5. In order to investigate the specific neurophysiological correlates of the SoA, spectral Power analyses were performed in the $\delta(1-4 \mathrm{~Hz}), \theta(4-8 \mathrm{~Hz}), \alpha(8-14 \mathrm{~Hz})$ and $\beta(14-30 \mathrm{~Hz})$ and $\gamma(30-40 \mathrm{~Hz})$ frequency bands. The statistical analyses aimed at determining if the recorded signal power was different between the conditions, i.e., Modulation vs. No-Modulation, for each of these frequency bands, at different time slots. Indeed, we separated the trials into 4 slots: (1) pre-trial (the $200 \mathrm{~ms}$ preceding the "GO" cue), (2) trial 


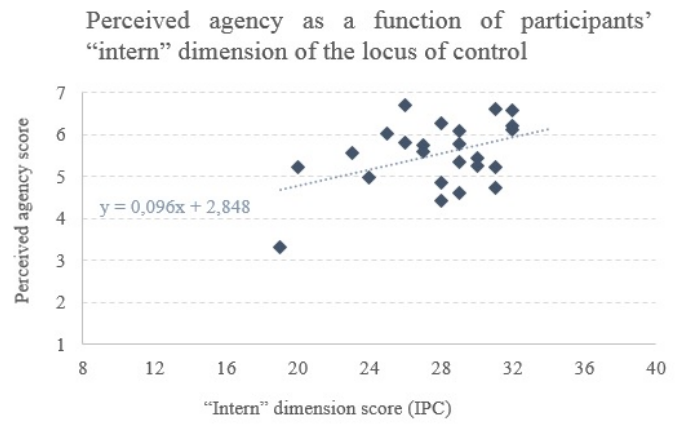

Feeling of immersion as a function of participants' "intern" dimension of the locus of control

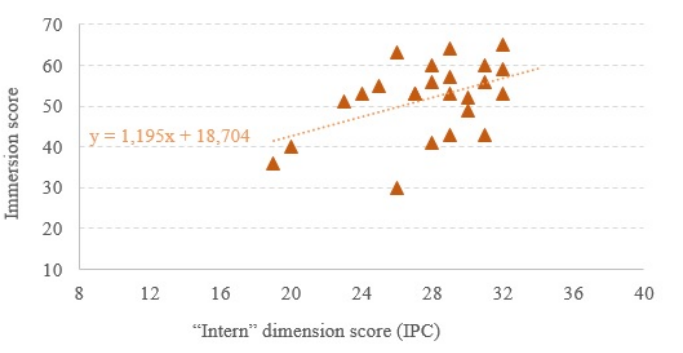

Figure 5. Diagrams representing: [up] the average score of perceived agency in non-manipulated trials (i.e., the basic level of agency - potentially ranging from 1 to 7 ) of each participant as a function of their score in the Intern dimension of the IPC questionnaire (potentially ranging from 8 to 40 ) [ $r=0.433, p \leq .05]$, together with the regression slope of equation $y=0.096 x+2.848[D(1,23)=5,077, p \leq .05]$; [down] the immersion score of each participant (computed from their responses to the 10 questions of the final questionnaire - potentially ranging from 10 to 70 ) as a function of their score in the Intern dimension of the IPC questionnaire $[r=0.473, p \leq .05]$, together with the regression slope of equation $y=1,195 x+18,704[D(1,23)=6,326, p \leq .05]$.

beginning ([0;500]ms after the "GO" cue), (3) feedback processing ([500-1000] after the "GO" cue) and (4) rest of the trial ([1000-7750] after the "GO" cue). The feeling of agency is supposed to occur during the trial beginning period while the judgment of agency should occur during the feedback processing period. These analyses were performed using permutation statistics, with a threshold p-value fixed at 0.05 , completed by a False Discovery Rate (FDR) correction for multiple comparisons. These analyses revealed significant differences between both conditions in the $\theta$ frequency band:

- during the trial beginning slot ([0;500]ms after the "GO" cue): over left fronto-central (FC1) and parietal (Pz, P3, PO3, PO7) areas $(\mathrm{p} \leq .05)$ - with a larger event-related synchronisation (ERS - i.e., increase of the signal power compared to baseline) in the no-Modulation condition (i.e., in high agency). This pattern might be specific to the feeling of agency.

- during the feedback processing slot ([500-1000]ms after the "GO" cue): over right temporal areas (C6) $(\mathrm{p} \leq .01)$ - with a greater ERS in the Modulation condition (i.e., in low agency). This pattern might be specific to the judgment of agency.

These results are depicted in Figure 6. To summarise, these analyses suggest that the SoA could be underlain by 2 neuromarkers, potentially reflecting each of the two components of the SoA, namely the feeling and the judgment of agency.

\subsection{Discussion}

This experiment aimed (1) at validating our approach to manipulate the SoA in a controlled VR experiment through the modulation of the 3 principles and (2) at validating the possibility to rely on specific neurophysiological markers, using EEG, to measure the SoA - and more specifically its two components. On the one hand, our results show that our attempt to manipulate the SoA was successful: indeed, the scores of perceived agency were significantly lower for all of the principle modulation-types compared to non-manipulated trials $(\mathrm{p} \leq .001)$. On the other hand, our results revealed specific neurophysiological patterns that seem to be consistent with the literature. Indeed, a stronger activation in the $\theta$ frequency band was measured at early stages of the trials over the left frontal areas when participants felt in control, while a stronger activation, also in the $\theta$ frequency band, was measured after the feedback was perceived and processed over the right parietal areas when participants did not feel in control. Given their timing as well as the brain structures they seem to be associated to, these neuromarkers could potentially reflect the two components of the SoA. More precisely, the frontal activation at early stages of the trials in high agency conditions might correspond to a stronger activation of the pre-motor cortex when participants experience a high feeling of agency. Also, the temporo-parietal activation later in the trials in low agency conditions could correspond to a stronger activation of the right posterior-parietal cortex when participants attribute the outcome to something/someone else then themselves, i.e., experience a low judgment of agency. Finally, our results suggest that participants' LoC influences significantly their basic level of agency as well as their feeling of immersion in the virtual environment. More precisely, the "intern" dimension score of the LoC enabled to explain more than $43 \%$ and $47 \%$ of the variance of participants' levels of agency and immersion, respectively. This result is compelling when considering that this is only one dimension of participants' personality and that so many other factors could take part in explaining modulations of the SoA in VR. This result stresses the importance of considering users' profile when trying to understand their level of agency, and maybe more generally their level of immersion.

\section{General discussion}

This study enabled us to validate the previously-introduced approaches of manipulation and measure of the SoA in a VR experiment. Twentyfour participants took part in a study during which they were embodied in a virtual avatar, in the first person perspective. They were asked to perform pre-determined movements and were provided with a visual feedback that was either manipulated (through the modulation of one of the three principles) or not manipulated. Results revealed that participants rated their SoA significantly lower in the manipulated than in the non-manipulated trials, whatever the principle modulated. It suggests that the three principles are relevant to be considered in VR. Indeed, the manipulations of the priority, exclusivity and consistency principles all induced a significant decrease of the SoA. The specific neurophysiological correlates of each of these manipulation types should now be further investigated. Interestingly enough, [3] had advocated that body ownership illusion could moderate the necessity of the exclusivity principle to be respected to experience a SoA. Our results (through the scores provided at the final questionnaire) show that participants felt immersed but felt significantly less in control when the exclusivity principle was modulated. Further analyses could provide insights on potential correlations between the feeling of immersion, of body-ownership and the effect of the exclusivity principle. In any case, this first result reinforces our hypothesis according to which the three principles are relevant and should be taken into account when investigating the SoA in VR. Second, it appeared that participants' profile, and more specifically the "Intern" dimension of the Locus of Control had an impact on their basic level of agency as well as on their feeling of immersion. Therefore, the user's profile should also be considered when investigating the factors impacting the SoA in VR. Third, the neurophysiological measures revealed the same patterns as the ones described in the literature. First, a stronger activation in the $\theta$ frequency-band was revealed at early stages of the trial when the participants felt in control, potentially reflecting a high feeling of agency. Second, a stronger activation in the same $\theta$ frequency-band was reported once the feedback was perceived when participants do not feel in control, potentially reflecting a low judgment of agency. The 

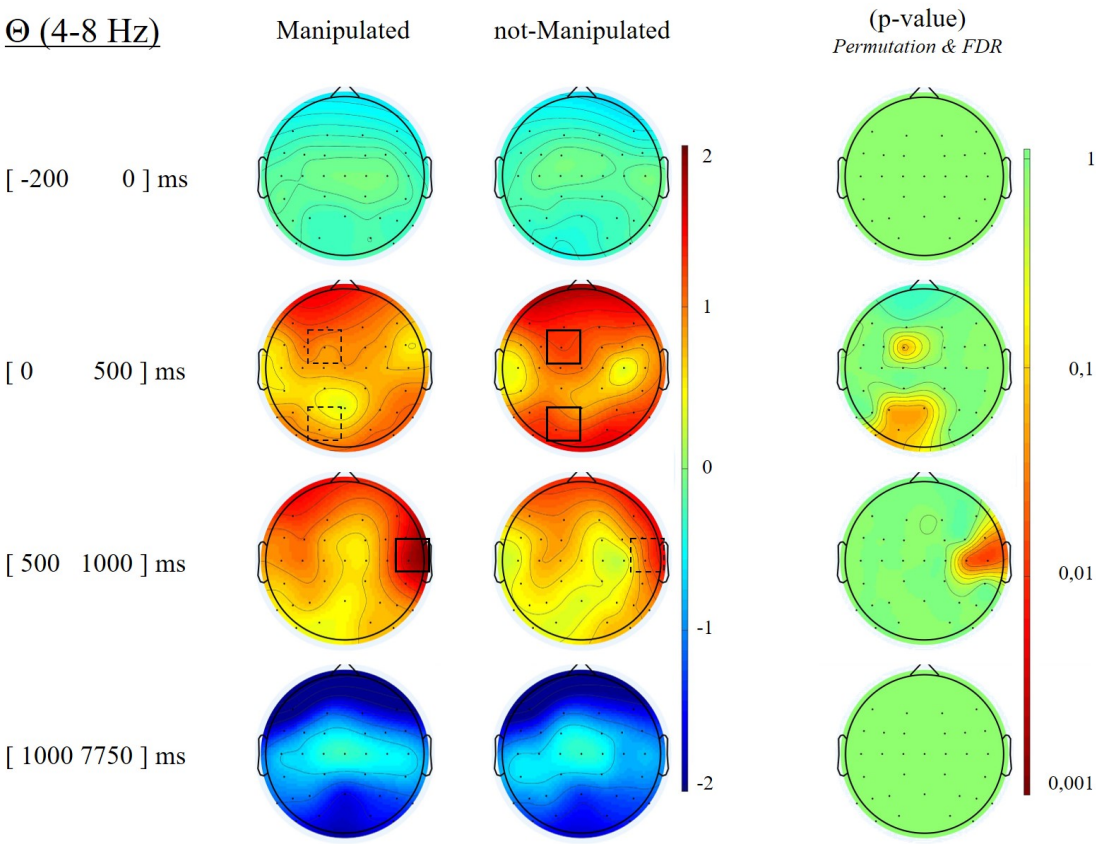

Figure 6. Spectral Power in the $\theta$ frequency band $(4-8 \mathrm{~Hz})$, for the different time slots and conditions (Manipulation vs. no-Manipulation of the sense of agency); $p$-values on the right indicate that after permutation statistics and FDR correction, this analysis revealed a stronger ERS in the no-Manipulation condition over the left fronto-central and parietal areas between 0 and $500 \mathrm{~ms}$ after the cue ( $p \leq .05)$, as well as a stronger ERS in the Manipulation condition over the right centro-temporal area between 500 and $1000 \mathrm{~ms}$ after the cue $(p \leq .05)$.

attribution of these EEG modulations to the feeling and judgment of agency specifically is only an hypothesis that should be further investigated in future works. The next step will consist in applying source reconstruction algorithms in order to determine more precisely the sources of the signal. Indeed, due to the poor spatial resolution of the EEG, we should stay cautious when inferring the precise location of the zones activated. Nevertheless, the strong differences between the high and low conditions as well as the apparent consistency between the literature and the obtained scalp maps is very encouraging. Also, this is the first time such correlates are revealed using surface EEG, thus offering extremely promising perspectives. Indeed, EEG is harmless, portable and is getting cheaper and cheaper with the emergence of EEG caps for the general public. Thus, the use of EEG in VR could be subject to a considerable expansion. The next challenge thus consists in determining if the highlighted neurophysiological markers could be used to perform on-line, i.e., real-time measures of the SoA. If that is the case, the EEG would enable a continuous monitoring of users' SoA and thus benefit the analysis and understanding of training approaches based on VR (e.g., one may hypothesise that in case of a cognitive-behavioural therapy of phobia, a low SoA of the patient in the VE might induce a low efficacy of the therapeutic approach). Also, for now, we are not able to compare the impact of each of the principles as we had no means to quantify the relative magnitude of the chosen modalities for each principle. If further investigations of the neurophysiological correlates of the SoA revealed a linear relationship between these markers (i.e., the fluctuations of amplitude of specific brain rhythms) and the SoA, it would provide an objective measure of the influence of each principle on the SoA. Notably, the priority principle is always slightly modulated as the feedback is provided in near real-time ( $\leq 50 \mathrm{~ms}$ latency) in non-manipulated trials. It would be interesting to assess the impact of this near real-time display on users' SoA and to determine the theoretical maximal SoA reachable as well as, more generally, a slope representing the SoA as a function of the lag. In addition, further neurophysiological analyses could enable us to determine the relationships between the two components and three principles of the SoA. For instance, the modulation of the different prin- ciples could be proved to alter differentially the feeling and judgment of agency. These elements would deeply improve our understanding of the SoA as well as provide powerful tools to evaluate the relevance of virtual environments and interaction paradigms, thus most likely leading to their improvement.

\section{CONCLUSION \& FUTURE WORK}

This paper provided four main contributions: (1) the characterisation of the SoA as a two-component process (divided into the feeling and the judgment of agency) which relies on three principles (the priority, consistency and exclusivity principles), (2) the proposition of an approach to manipulate the SoA in virtual environments through the modulation of its three principles, (3) the proposition of an approach to measure the SoA, and more specifically its two components, using surface EEG and finally (4) the validation of these approaches to manipulate and measure the SoA thanks to an experiment ( $\mathrm{N}=24$ participants).

By way of a conclusion, it can be stated that by bringing forward the literature from different research fields we could be provided with promising new approaches to investigate and deepen our understanding of the SoA in VR. Our paper provides a first contribution to this end. We provided a new perspective to apprehend the different dimensions of the SoA, and to manipulate and measure the SoA in virtual environments. As stated in the discussion, further investigations are required first to evaluate how and how much each of the principles influences the SoA, and second to assess the suitability of the neurophysiological markers to be used for real-time and continuous monitoring of the SoA.

In the long term, this work could benefit different applications. In the field of video-games for instance, the on-line monitoring of the SoA could be used to test the quality and relevance of a virtual environment and of its associated interaction paradigm relatively to the principles of priority, consistency and exclusivity. But this work could also be of interest for therapeutic applications. Indeed, a high $\theta$-power in right temporo-parietal areas has been revealed during auditory-hallucinations in schizophrenic patients [6]. This region being typically activated when one does not feel in control, this pattern could at least in part explain why schizophrenic patients tend to attribute these hallucinations as 
manifestations of the fact that other people or forces would control them. Using on-line monitoring of the SoA and of this marker during cognitive-behavioural treatments might help to detect, explain and manage these patients' hallucinations. These two examples reflect the wide range of applications that could benefit from this work.

\section{ACKNOWLEDGMENTS}

The authors would like to thank Jussi Lindgren for his help regarding the neurophysiological analyses. This work was supported in part by the Inria/EPFL International Lab.

\section{REFERENCES}

[1] F. Argelaguet, L. Hoyet, M. Trico, and A. Lécuyer. The role of interaction in virtual embodiment: Effects of the virtual hand representation. In Virtual Reality (VR), pp. 3-10, 2016.

[2] S. Arzy, G. Thut, C. Mohr, C. M. Michel, and O. Blanke. Neural basis of embodiment: distinct contributions of temporoparietal junction and extrastriate body area. Journal of Neuroscience, 26(31):8074-8081, 2006

[3] D. Banakou and M. Slater. Body ownership causes illusory self-attribution of speaking and influences subsequent real speaking. Proceedings of the National Academy of Sciences, 111(49):17678-17683, 2014.

[4] O. Blanke and T. Metzinger. Full-body illusions and minimal phenomenal selfhood. Trends in cognitive sciences, 13(1):7-13, 2009.

[5] O. Blanke, M. Slater, and A. Serino. Behavioral, neural, and computational principles of bodily self-consciousness. Neuron, 88(1):145-166, 2015.

[6] J. N. Booth and M. A. Persinger. Discrete shifts within the theta band between the frontal and parietal regions of the right hemisphere and the experiences of a sensed presence. The Journal of neuropsychiatry and clinical neurosciences, 21(3):279-283, 2009.

[7] M. Botvinick and J. Cohen. Rubber hands' feel'touch that eyes see. Nature, 391(6669):756, 1998.

[8] V. Chambon, D. Wenke, S. M. Fleming, W. Prinz, and P. Haggard. An online neural substrate for sense of agency. Cerebral Cortex, 23(5):10311037, 2012.

[9] N. David, B. H. Bewernick, M. X. Cohen, A. Newen, S. Lux, G. R. Fink, N. J. Shah, and K. Vogeley. Neural representations of self versus other: visual-spatial perspective taking and agency in a virtual ball-tossing game. Journal of cognitive neuroscience, 18(6):898-910, 2006.

[10] N. David, A. Newen, and K. Vogeley. The sense of agency and its underlying cognitive and neural mechanisms. Consciousness and cognition, 17(2):523-534, 2008.

[11] F. De Vignemont. Embodiment, ownership and disownership. Consciousness and cognition, 20(1):82-93, 2011.

[12] H. G. Debarba, E. Molla, B. Herbelin, and R. Boulic. Characterizing embodied interaction in first and third person perspective viewpoints. In $3 D$ User Interfaces, 2015 IEEE Symposium on, pp. 67-72, 2015.

[13] A. Delorme and S. Makeig. Eeglab: an open source toolbox for analysis of single-trial eeg dynamics including independent component analysis. Journal of neuroscience methods, 134(1):9-21, 2004.

[14] H. H. Ehrsson, C. Spence, and R. E. Passingham. That's my hand! activity in premotor cortex reflects feeling of ownership of a limb. Science, 305(5685):875-877, 2004.

[15] C. Farrer and C. D. Frith. Experiencing oneself vs another person as being the cause of an action: the neural correlates of the experience of agency. Neuroimage, 15(3):596-603, 2002.

[16] S. Gallagher. Philosophical conceptions of the self: implications for cognitive science. Trends in cognitive sciences, 4(1):14-21, 2000.

[17] S. Gallagher. Multiple aspects in the sense of agency. New Ideas in Psychology, 30(1):15-31, 2012.

[18] M. Gonzalez-Franco, A. I. Bellido, K. J. Blom, M. Slater, and A. Rodriguez-Fornells. The neurological traces of look-alike avatars. Frontiers in human neuroscience, 10, 2016.

[19] P. Haggard. Sense of agency in the human brain. Nature Reviews Neuroscience, 18(4):196-207, 2017.

[20] A. Harris. Harris tests of lateral dominancepsychological corporation. New York, 1958.

[21] C. Jeunet, B. NKaoua, and F. Lotte. Advances in user-training for mentalimagery-based bci control: Psychological and cognitive factors and their neural correlates. Progress in brain research, 228:3-35, 2016.

[22] A. Kalckert and H. H. Ehrsson. Moving a rubber hand that feels like your own: a dissociation of ownership and agency. Frontiers in human neuroscience, 6, 2012.
[23] S. Y. Kang, C.-H. Im, M. Shim, F. B. Nahab, J. Park, D.-W. Kim, J. Kakareka, N. Miletta, and M. Hallett. Brain networks responsible for sense of agency: an eeg study. PloS one, 10(8):e0135261, 2015.

[24] K. Kilteni, R. Groten, and M. Slater. The sense of embodiment in virtual reality. Presence: Teleoperators and Virtual Environments, 21(4):373-387, 2012.

[25] K. Kilteni, J.-M. Normand, M. V. Sanchez-Vives, and M. Slater. Extending body space in immersive virtual reality: a very long arm illusion. PloS one, 7(7):e40867, 2012.

[26] E. Kokkinara, K. Kilteni, K. J. Blom, and M. Slater. First person perspective of seated participants over a walking virtual body leads to illusory agency over the walking. Scientific reports, 6:28879, 2016.

[27] E. Kokkinara, M. Slater, and J. López-Moliner. The effects of visuomotor calibration to the perceived space and body, through embodiment in immersive virtual reality. ACM Transactions on Applied Perception (TAP), 13(1):3, 2015.

[28] S. Kühn, M. Brass, and P. Haggard. Feeling in control: Neural correlates of experience of agency. Cortex, 49(7):1935-1942, 2013.

[29] B. Lenggenhager, P. Halje, and O. Blanke. Alpha band oscillations correlate with illusory self-location induced by virtual reality. European Journal of Neuroscience, 33(10): 1935-1943, 2011.

[30] H. Levenson. Activism and powerful others: Distinctions within the concept of internal-external control. Journal of personality assessment, 38(4):377-383, 1974.

[31] S. A. Linkenauger, M. Leyrer, H. H. Bülthoff, and B. J. Mohler. Welcome to wonderland: The influence of the size and shape of a virtual hand on the perceived size and shape of virtual objects. PloS one, 8(7):e68594, 2013.

[32] C. D. Murray, J. Fox, and S. Pettifer. Absorption, dissociation, locus of control and presence in virtual reality. Computers in Human Behavior, 23(3):1347-1354, 2007.

[33] F. B. Nahab, P. Kundu, C. Gallea, J. Kakareka, R. Pursley, T. Pohida, N. Miletta, J. Friedman, and M. Hallett. The neural processes underlying self-agency. Cerebral Cortex, 21(1):48-55, 2010.

[34] N. H. Nie, D. H. Bent, and C. H. Hull. Spss: Statistical package for the social sciences. Technical report, McGraw-Hill New York, 1970.

[35] G. Padrao, M. Gonzalez-Franco, M. V. Sanchez-Vives, M. Slater, and A. Rodriguez-Fornells. Violating body movement semantics: Neural signatures of self-generated and external-generated errors. Neuroimage, 124:147-156, 2016.

[36] Y. Renard, F. Lotte, G. Gibert, M. Congedo, E. Maby, V. Delannoy, O. Bertrand, and A. Lécuyer. Openvibe: an open-source software platform to design, test, and use brain-computer interfaces in real and virtual environments. Presence: teleoperators \& virtual environments, 19(1):35-53, 2010.

[37] M. V. Sanchez-Vives and M. Slater. From presence to consciousness through virtual reality. Nat Rev Neurosci, 6(4):332-339, 2005.

[38] M. Sperduti, P. Delaveau, P. Fossati, and J. Nadel. Different brain structures related to self-and external-agency attribution: a brief review and meta-analysis. Brain Structure and Function, 216(2):151-157, 2011.

[39] M. Synofzik, G. Vosgerau, and A. Newen. Beyond the comparator model: a multifactorial two-step account of agency. Consciousness and cognition, 17(1):219-239, 2008.

[40] G. Tieri, E. Tidoni, E. Pavone, and S. Aglioti. Mere observation of body discontinuity affects perceived ownership and vicarious agency over a virtual hand. Experimental brain research, 233(4):1247-1259, 2015.

[41] M. Tsakiris, G. Prabhu, and P. Haggard. Having a body versus moving your body: How agency structures body-ownership. Consciousness and cognition, 15(2):423-432, 2006.

[42] K. Vogeley, M. May, A. Ritzl, P. Falkai, K. Zilles, and G. R. Fink. Neural correlates of first-person perspective as one constituent of human selfconsciousness. Journal of cognitive neuroscience, 16(5):817-827, 2004.

[43] D. M. Wegner. Précis of the illusion of conscious will. Behavioral and Brain Sciences, 27(5):649-659, 2004.

[44] D. M. Wegner, B. Sparrow, and L. Winerman. Vicarious agency: experiencing control over the movements of others. Journal of personality and social psychology, 86(6):838, 2004.

[45] D. M. Wegner and T. Wheatley. Apparent mental causation: Sources of the experience of will. American psychologist, 54(7):480, 1999.

[46] A. C. Wellerdiek, M. Leyrer, E. Volkova, D.-S. Chang, and B. Mohler. Recognizing your own motions on virtual avatars: is it me or not? In Proceedings of the ACM Symposium on Applied Perception, p. 138, 2013. 